\title{
AEROPUERTOS INTELIGENTES: ACEPTACIÓN DE LA TECNOLOGÍA POR PARTE DE LOS PASAJEROS
}

\author{
Mónica Monge Zamorano* \\ Universidad Politécnica de Madrid \\ https://orcid.org/0000-0003-3403-2898 \\ María Cristina Fernández-Laso** \\ Universidad Rey Juan Carlos. Madrid \\ https://orcid.org/0000-0001-5719-8888 \\ Javier de Esteban Curiel*** \\ Universidad Rey Juan Carlos. Madrid \\ https://orcid.org/0000-0001-9735-286X
}

\section{RESUMEN}

En este trabajo se analiza la aceptación de las tecnologías que hacen de un aeropuerto un Aeropuerto Inteligente, por parte de los pasajeros españoles. En concreto, se comprueba cómo el uso de tecnologías autoservicio en los procesos de embarque, obtención de etiquetas de equipaje y facturación del equipaje aumenta la satisfacción del pasajero. También se muestra que el deseo de utilización de las nuevas tecnologías en estos procesos y del uso de videojuegos y pasaporte biométrico está relacionada con la edad de los pasajeros.

Palabras clave: Aeropuertos inteligentes; tecnología; pasajero; edad.

\section{Smart airports: Acceptance of technology by passengers}

\section{ABSTRACT}

This paper analyzes the acceptance of the technologies that make an airport an Smart Airport, by Spanish passengers. In particular, it is verified how the use of self-service tech-

Fecha de recepción: 14 de noviembre de 2018.

Fecha de aceptación: 20 de febrero de 2019.

* Departamento de Ingeniería de Organización, Administración de Empresas y Estadística. Escuela Técnica Superiorde Ingeniería Aeronáutica y de Espacio. Universidad Politécnica de Madrid. Plaza del Cardenal Cisneros, 3. 28040 MADRID (España).E-mail: monica.monge@upm.es

** Departamento de Economía de la Empresa.Universidad Rey Juan Carlos. Paseo de los Artilleros, s/n. 28032 MADRID (España).E-mail: cristina.fernandez@urjc.es

*** Departamento de Ciencias de la Educación, Lenguaje, Cultura y Artes; Ciencias Histórico-Jurídicas y Humanísticas y Lenguas Modernas. Universidad Rey Juan Carlos. Paseo de los Artilleros, s/n. 28032 MADRID (España).E-mail: javier.deesteban@urjc.es 
nologies in the boarding, obtaining luggage tags and baggage check processes increases the passenger's satisfaction. It also shows that the desire to use new technologies in these processes and the use of video games and biometric passports is related to the age of the passengers.

Keywords: Smart airports; technology; passenger; age.

\section{INTRODUCCIÓN}

La investigación que da origen al presente documento se enmarca dentro del campo del turismo inteligente y tiene su origen en dos fenómenos: el auge de las Tecnologías de la Información y la Comunicación (TIC) y la aparición de nuevas tecnologías como la Comunicación de Campo Cercano, la tecnología de Código de Barras, la Identificación por Radiofrecuencia, las tecnologías de geolocalización, la Realidad inmersiva, los sistemas biométricos, la Inteligencia Artificial y la Robótica y el Blockchain o Tecnología de Bloques, y la creciente importancia de la actividad turística, concretamente la aeroportuaria.

La importancia de la industria aeroportuaria viene avalada por los datos: a nivel mundial, en 2016 los turistas que viajaron en avión gastaron alrededor de 650.000 millones de dólares, el ingreso medio por pasajero y kilómetro de la industria creció un 7,4 \% y el número de conexiones entre pares de ciudades superó las 18.200 , alrededor de 500 más de las ofrecidas en 2015 y casi el doble que hace 20 años. La aviación mantiene 67,7 millones de puestos de trabajo en su cadena de suministro(IATA, 2017).

España ocupó en 2017 el segundo lugar del mundo en llegadas de turistas internacionales, superada únicamente por Francia y seguida de Estados Unidos. Los aeropuertos son la principal vía de entrada de los turistas que visitan España. Más de 66,6 millones (el $81,5 \%$ ) utilizaron el avión como medio de transporte, el 15,7\% se desplazó por carretera y menos del $3 \%$ en otros tipos de transporte. España recibió 81,8 millones de turistas internacionales en 2017, 6,2 millones más que el año anterior (un 8,6\% de variación interanual), lo que supone un nuevo récord histórico por quinto año consecutivo(Aena, 2018). La incorporación de las TIC en el ámbito empresarial puede resultar un elemento clave para mejorar la competitividad, impulsar el crecimiento económico y lograr una mayor creación de empleo (Eguía y Alonso, 2002).

El aerotropolis, entendido como una ciudad con un aeropuerto como núcleo y extensiones adyacentes de servicios empresariales relacionados con la aviación y sus áreas residenciales y recreativas correspondientes(Kasarda, 2000), también se beneficia de la implantación de las nuevas tecnologías.

Hasta el momento abundan en la literatura científica tanto los trabajos sobre ciudades inteligentes (Buhalis y Amaranggana, 2013; Cohen, 2012; Vanolo, 2013)y actividad aeroportuaria como sobre las TIC y la relación de su uso con la edad; sin embargo es verdaderamente escasa la investigación de la aceptación de las tecnologías en los aeropuertos y no se ha considerado en la literatura existente el aeropuerto inteligente. Este artículo trata de cubrir ese hueco en un ámbito de tanta relevancia.

Los avances tecnológicos han permitido históricamente a empresas e instituciones ofrecer nuevos productos y servicios y reducir costes. Basta con pensar en algunos hitos 
tales como el telar automático, la máquina de vapor, el ordenador personal o el teléfono móvil. La tecnología comenzó a introducirse en la industria antes que en el sector servicios por sus ventajas en cuanto a aumento de la productividad y ahorro de costes. Según Inglada y Coto-Millán (2017) los aeropuertos españoles experimentaron entre los años 1992 y 2012 un moderado incremento de la productividad total de los factores que es imputable prácticamente en exclusiva al crecimiento de la eficiencia técnica. Sin embargo, es conocido que, a pesar de sus ventajas, cualquier innovación técnica, como las anteriormente mencionadas, se enfrenta en los primeros estadios de su implantación a resistencias por parte de los diferentes grupos que participan en la actividad: desde los directivos a los consumidores o usuarios, pasando por el personal que interviene en la fabricación del producto o la prestación del servicio. Durante la primera década del siglo XXI, autores como Ha et al. (2011) y Sinkovics et al. (2002) estudiaron la tecnofobia, su medición y distintas formas de evitarla (Chukhray, 2012).

Distintas instituciones han recomendado el uso de estas tecnologías (OACI, 2015). Los miembros de la Airports Council International (ACI) reconocen los beneficios del uso de la biometría para confirmar la identidad personal en el control de fronteras y en él recomiendan la implementación de sistemas biométricos en los aeropuertos para simplificar, agilizar y mejorar el proceso de viaje del pasajero, incluyendo controles de fronteras y seguridad, al tiempo que se reducen costes (ACI, 2005, ACI, 2009).

El presente artículo tiene pues, como objetivo,conocer la aceptación de las distintas tecnologías que hacen de un aeropuerto un aeropuerto inteligente, en concreto, las relaciones entre el uso de éstasy la satisfacción del pasajero en los procesos de embarque, obtención de etiquetas de equipaje y facturación del mismo, y la relación de la edad con la aceptación de estas tecnologías. Para ello, el presente artículo se articula en cuatro partes: tras la presente introducción se explora la literatura científica existente, que se ha abordado mediante la aproximación a las tecnologías de utilidad en los aeropuertos y a la aceptación de las tecnologías por parte de los usuarios, lo cual permite la formulación de las hipótesis de trabajo:

- $\mathrm{H}_{1}$ : La utilización de tecnología en el aeropuerto aumenta la satisfacción del pasajero

- $\mathrm{H}_{2}$ : La edad está relacionada con la aceptación de las tecnologías en los aeropuertos

Posteriormente, se aborda la metodología llevada a cabo centrada en una encuesta a 400 personas; se exponen los resultados principales del estudio y en el último bloque, se desarrollan las conclusiones del trabajo.

\section{LA TECNOLOGÍA EN LOS AEROPUERTOS: DEL DESTINO INTELIGENTE AL AEROPUERTO INTELIGENTE}

El concepto de Destino Turístico Inteligente surge del desarrollo de las Ciudades Inteligentes. Con la tecnología integrada en todas las organizaciones y entidades, los destinos podrán explotar las sinergias entre tecnología y componente social para enriquecer la experiencia turística. El turismo inteligente implica la aplicación de tecnologías inteligentes en todas las fases del viaje (Koo et al., 2016).Aprovechar las nuevas tecnologías e Internet 
permite a los destinos mejorar su competitividad mediante el aumento de su visibilidad, reduciendo los costos y mejorando la cooperación local. Aplicando el concepto de inteligencia a la satisfacción de las necesidades de los viajeros antes, durante y después de su viaje, los destinos podrán incrementar su competitividad (Buhalis y Amaranggana, 2013).

Desde el punto de vista urbanístico, en 1997 surge el estudio "Project CITIES" en Filadelfia, en la Universidad de Pennsylvania, consistente en observar las experiencias urbanas en veinte ciudades innovadoras de los cinco continentes. Fue a raíz de este proyecto que se bautizaron como smart places o territorios inteligentes a aquellas ciudades innovadoras capaces de encontrar un equilibrio entre cohesión y desarrollo social, competitividad económica, y sostenibilidad ambiental y cultural, el precedente más cercano a las smartcities (Vergara y De las Rivas, 2004).

Existen múltiples definiciones de ciudad inteligente (Boulanger y Jeannin, 2016; Caragliu, Del Bo y Nijkamp, 2011; Komninos, Pallot y Schaffers, 2013; Nam y Pardo, 2011; SEGITTUR, 2015); todas ellas comparten la idea de que la tecnología al servicio del ciudadano hace de la ciudad una ciudad inteligente.Debido a la amplitud de estas definiciones, ha habido diversos intentos de evaluar el grado de inteligencia de estas ciudades. Entre ellos, destacan el llevado a cabo en el 2012 por la International Data Corporation (IDC) para elaborar el Índice de Ciudades Inteligentes de ciudades españolas con una población superior a los 150.000 habitantes elaborado a partir de parámetros de inteligencia (infraestructuras, movilidad, políticas medioambientales y servicios) y de las fuerzas habilitadoras (la economía los ciudadanos y las Tecnologías de la Información y la Comunicación) (IDC, 2012) y la Rueda de la Ciudad Inteligente, como una herramienta para el desarrollo y seguimiento de las estrategias de las Ciudades Inteligentes (Cohen, 2012).

Por tanto, se puede definir el "Aeropuerto Inteligente" como el aeropuerto que pone la tecnología al servicio del usuario para aumentar la eficiencia de servicio, la sostenibilidad medioambiental y la comodidad y satisfacción del pasajero. Un aeropuerto Inteligente es una parte vital de la Ciudad Inteligente.

Boyd Cohen desarrolló la Rueda de la Ciudad Inteligente como una herramienta para el desarrollo y seguimiento de las estrategias de las Ciudades Inteligentes (Cohen, 2012). Cohen define algunos indicadores para cada una de las dimensiones inteligentes, que podemos aplicar al aeropuerto inteligente:

a) Gestión Inteligente. Se refiere a la transparencia en la gestión mediante sistemas de modernización de su administración con open data y participación ciudadana

b) Medio Ambiente Inteligente, relacionado con la optimización energética que conduce a la gestión sostenible de los recursos disponibles

c) Movilidad Inteligente, referida a la accesibilidad, tanto en el interior como fuera, y la disponibilidad de modernos sistemas de transporte

d) Economía Inteligente, relacionada con la implementación de estrategias económicas basadas en la tecnología digital

e) Personas Inteligentes, ligado a la cualificación del capital humano

f) Vida Inteligente, engloba la calidad de vida medida en términos de entorno saludable, cohesión social, atracción turística y disponibilidad de servicios 
Un aeropuerto constituye la puerta de entrada a un territorio, es un icono que desde su propia denominación ya encierra una referencia espacial (Serrano, Espejo y García Marín, 2016).Con un de tren o los puertos, el aeropuerto no es un destino para los viajeros; es un punto de transición (Foodness y Murray, 2007). Pero el aeropuerto ofrece algunas características únicas, como el mayor tiempo que pasan los viajeros en él -alrededor de una hora en el aeropuerto una vez que han entrado en la terminal (WorldAirportWeek, 2000 en Fodness y Murray 2007) - debido a los procesos de control de seguridad y embarque, y el tener siempre un control de aduana, aunque no sea necesario para todos los pasajeros. Augé (1993) entiende los aeropuertos como "no lugares" donde se reinscribe sin cesar el juego intrincado de la identidad y de la relación. Si un lugar puede definirse como lugar de identidad, relacional e histórico, un espacio que no puede definirse ni como espacio de identidad ni como relacional ni como histórico, definirá un no lugar. Internet y las aplicaciones móviles podrían convertirlos en "lugares", puesto que acaban con el anonimato de los pasajeros. Con un diseño adecuado, un aeropuerto moderno es un glamuroso símbolo de modernidad, que puede diferenciar una ciudad de sus rivales y validar su "Inteligencia" (Huston, 2015).

Los lugares se han convertido en "un artículo comercial que se ha de promocionar y vender para atraer un capital, unos inversores, unas personas y unos turistas que están en movimiento"(Hall, 2009, p. 134).El aeropuerto es un espacio físico con una serie de servicios tangibles o intangibles que Bitner (1992) bautizó como servicescape; un espacio holístico donde las señales, símbolos, instrumentos y personas interactúan como un todo. Los usuarios de un aeropuerto son variados, incluyendo pasajeros, aerolíneas, empleados, restauradores, y otros. Este estudio está enfocado en el pasajero como usuario final de los servicios aeroportuarios. El comportamiento y las expectativas de los pasajeros dependen del tipo de viajero, el propósito de su viaje y sus circunstancias personales. Pero a pesar de estas diferencias, todos los pasajeros están en el aeropuerto con el único propósito de cambiar de medio de transporte. El aeropuerto no es un destino para los pasajeros, es un punto de transición.Aunque existan distintos tipos de pasajeros con diferentes motivos para viajar, todos ellos están ahí con el único propósito de pasar de un medio de transporte terrestre a uno aéreo. Al igual que otros intercambiadores de transporte, como las estaciones

Los aeropuertos han sido entendidos históricamente como lugares donde operan los aviones, incluyendo pistas, hangares, torres de control, terminales y otras instalaciones al servicio de las aeronaves, pasajeros y carga.Este concepto ha dado paso a uno más amplio, ya que además de su infraestructura y servicios aeronáuticos centrales, prácticamente todos los aeropuertos principales han desarrollado cada vez más instalaciones y servicios comerciales no aeronáuticos. El modelo de Ciudad Aeroportuaria (o Aerotropolis) se está convirtiendo en el nuevo modelo de gestión y desarrollo internacional. Aeropuertos como los de Amsterdam, Zurich o Beijing han adoptado este modelode planificación para desarrollar sus terminales y áreas terrestres como un medio fundamental para financiar las operaciones aeroportuarias y contribuir a sus propios negocios competitivos.(Kasarda, 2000).

La implantación de dispositivos y tecnologías autoservicio en los aeropuertos se lleva a cabo por las aerolíneas y el gestor aeroportuario. En España la propiedad de los aeropuertos y todo lo relacionado con la responsabilidad en cuanto a gestión y operación de 
los mismos recae en el ente autónomo estatal Aeropuertos Españoles y Navegación Aérea (Aena) (Ruiz, Benito; Pindado Carrión, 2012), por tanto, no existen grandes diferencias en este sentido entre grandes hubs y aeropuertos regionales.

\section{EL PASAJERO}

\subsection{Cambios en la relación entre el aeropuerto y el pasajero}

Los aeropuertos eran considerados tradicionalmente como espacios públicos cuya finalidad era proporcionar traslados rápidos y seguros (Florido-Benítez, 2016). Los pasajeros llegan a un aeropuerto como resultado de un proceso de toma de decisiones limitado habitualmente a la combinación de rutas aéreas, precios y horarios que ofrecen las aerolíneas, y su localización. En la mayoría de los casos, la elección del aeropuerto está determinada por la decisión de las aerolíneas de dónde operar (Graham, 2013).Sin embargo, a partir de los años ochenta, laliberalización de las aerolíneas y otros sectores de la industria aeroportuaria hizo que los aeropuertos comenzaran a competir por las rutas aéreas y a introducir el marketing para proteger o incrementar su cartera de aerolíneas clientes (Forsyth et al., 2010).

Al convertirse los pasajeros en consumidores cada vez más sofisticados y exigentes, los aeropuertos creyeron que podrían influir en las decisiones de las aerolíneas dirigiendo sus estrategias de marketing al usuario final, ofreciendo mejores servicios o la promesa de excelentes niveles de satisfacción del consumidor. En los años noventa, con la irrupción de las aerolíneas low-cost, la industria aeroportuaria se volcó en la calidad de servicio como una estrategia para lograr ventaja competitiva (Lee-Mortimer, 1993). Un estudio de Camisón et. al (1997) demuestra que es a partir de 1991 cuando crece espectacularmente el número de artículos a nivel internacional sobre calidad en turismo. Sin embargo, revela una gran concentración de la investigación en las actividades de alojamiento y restauración y un casi total olvido del sector de transporte: entre 1986 y 1996 sólo se recogen dos artículos sobre gestión de calidad en este sector. En este contexto aparece el Modelo SERVQUAL, propuesto por Parasuraman et. al (1985) que consideraba cinco dimensiones de medición de la calidad del servicio, a saber: fiabilidad, capacidad de respuesta, tangibilidad, seguridad y empatía. Existen numerosos trabajos que utilizan la metodología SERVQUAL para medir la satisfacción de los pasajeros en los aeropuertos (Robledo, 1997; Chau y Kao, 2009; Gilbert y Wong, 2003; Sultan, Farena y Simpson, 2000), pero no evalúan el impacto de la tecnología en la percepción de calidad de servicio.

Fodness y Murray (2007) indicaron en su trabajo sobre las expectativas de los pasajeros que los estudios sobre medición de calidad de servicio en aeropuertos son escasos, pero que plantean un nuevo aporte para la industria, pues identifican las características de los aeropuertos de Estados Unidos, permitiendo medir la preferencia del pasajero de un aeropuerto frente a otro. Trabajos como los de Rhoades et. al (2000), Chen (2002) y Yeh y Kuo (2003) consideraron la percepción del consumidor a través de la visión de los operadores de los aeropuertos.Fodness, y Murray (2007) criticaron este enfoque consultando directamente a los pasajeros. A partir de entonces, otros estudios han seguido esta línea, por ejemplo, Tsai, Hsu y Chou (2011), Liou et al. (2011) o Pantouvakis y Renzi (2016), que proponen un 
modelo en el que la calidad de servicio en los aeropuertos se evalúa con cuatro dimensiones: servicescape, señalización, servicio e imagen (tampoco tecnología) y concluyeron que la nacionalidad de los pasajeros influye en su percepción de la calidad de los aeropuertos.

Internet y otras nuevas tecnologías juegan un papel fundamental en las estrategias de marketing de los aeropuertos, reduciendo el tiempo de espera de los pasajeros en los distintos procesos a llevar a cabo en el aeropuerto, proporcionando mayor seguridad personal, de su equipaje y sus automóviles, mejorando la información proporcionada por los distintos agentes aeroportuarios, e incluso proporcionando oportunidades de ocio en su estancia en el aeropuerto.

\subsection{Aceptación de la tecnología}

La primera y última percepción que un pasajero tiene de un destino turístico tiene lugar en el aeropuerto. Por esto, la satisfacción del pasajero en el aeropuerto es una de las formas de aumentar la ventaja competitiva tanto del propio aeropuerto, como del destino turístico próximo a éste(Rendeiro, 2006).

Internet y otras TIC han cambiado el comportamiento del consumidor. Han aumentado la eficiencia de las reservas y pagos, y progresivamente, los viajeros las han ido utilizando para obtener información más segura, rica y personalizada de todas las fases de su viaje (Huang, Goo, Nam y Yoo, 2017).Existen numerosas aportaciones tecnológicas para el lado aire de un aeropuerto (Ceylan et al. 2015), pero el pasajero no las percibe directa e inmediatamente. Es por ello que este trabajo se centra en el lado tierra.

Paralelamente, han aparecido nuevas tecnologías que han permitido crear otros tantos dispositivos que hacen de un aeropuerto un aeropuerto inteligente. Estas tecnologías han surgido en distintos momentos del tiempo y han tenido un desigual desarrollo e implantación.

Quizá la más antigua sea la biometría, ya que en 1900 Scotland Yard ya utilizaba la huella dactilar para confirmar la identidad de los sospechosos. El reconocimiento del iris se hace realidad en 1985 con la patente de Flom y Safir ${ }^{(1985)}$.Biometría es un término genérico usado para referirse a las características físicas o conductuales que se pueden medir para verificar la identidad de un individuo. La biometría física mide una parte de la anatomía del individuo, por ejemplo, la huella dactilar, la cara, la mano y el iris; la biometría conductual mide una acción, por ejemplo, la voz, la firma; en ambos casos las características deben ser identificables, universales, únicas y permanentes (ACI, 2005). Los sistemas biométricos informáticos funcionan en dos fases: en la primera etapa de inscripción, un lector biométrico registra y captura las características físicas o conductuales de una persona debidamente identificada y los introduce en una base de datos para su registro, comprobación y almacenamiento. En fase de identificación, el mismo lector biométrico registra y captura las características físicas o conductuales de una muestra no identificada, las compara con la base de datos por medio de algoritmos y determina o no correlación, es decir, identidad (Tapiador, Marino y Sigüenza, 2005).El lanzamiento de la primera prueba biométrica en 2015 por KLM en el aeropuerto de Aruba demostró que la biometría es una tecnología clave para reducir a minutos los tiempos de los procesos en los aeropuertos y hacerlos más transparentes, seguros y sin interrupciones (Hosteltur, 2017). 
En 1952 surge la tecnología de código de barras, producto de la implementación de un trabajo de master en la Escuela de Negocios de la universidad de Harvard en 1932 (Woodland y Bernard, 1952). El sistema consiste en una serie de líneas y espacios de distintos anchos, que almacenan la información que es leída por un dispositivo que captura esta información en forma de códigos. Su incorporación se remonta a 1967 en Cincinnati (USA), donde se probó por primera vez en un supermercado (Seideman, 1993). Actualmente es eles el tipo de sistema de identificación automática de objetos más difundido en el mundo.

La tecnología de Identificación por Radiofrecuencia (RFID) se utiliza para transmitir señales sin cable y sin la presencia física del objeto que se desea identificar, y para automatizar el seguimiento de objetos usando la información de una base de datos centralizada, de una forma muy fácil y sencilla. Las etiquetas RFID se colocan en los diversos objetos que se desee seguir o identificar mediante la información almacenada en ellas. Estos objetos son detectados por los lectores de RFID al emitir las ondas electromagnéticas que estarán presentes alrededor del objeto móvil. Aunque las aplicaciones comerciales de RFID datan de los años 60, su uso se generalizó a partir del 2000 (Tajima, 2007).

En 1994 Paul Milgram y FumioKishino introdujeron el término Realidad Mixta (RM), donde la Realidad Aumentada (RA) es una parte de la RM (Milgram et al., 1994). La diferencia entre RA y Realidad Virtual (RV) es que en la primera, se añade información digital a imágenes y contextos de la vida real, mientras que la segunda ofrece al usuario un nuevo mundo en el que puede sumergirse (Avelar y Santos, 2014). La RV crea una experiencia inmersiva (Rebelo, Noriega, Duarte, 2012). Aunque el aspecto visual de la RV suele captar la mayor atención, un elemento de audio también puede ser muy importante para la creación de entornos virtuales realistas. Es importante considerar la direccionalidad, ya que los sonidos pueden provenir de diferentes puntos del entorno virtual(Mario Gutierrez y Vexo, 2008), sensaciones con “dispositivos táctiles" como guantes para reproducir la sensación de hacer fuerza, así como señales térmicas y presión que imitan el peso de un objeto (Guttentag, 2010), la simulación olfativa y de saborespuede incrementar la sensación de presencia del usuario.

La Comunicación de Campo Cercano (NFC) es una tecnología de transmisión basada en el estándar de tarjetas inteligentes sin contacto, con una distancia máxima de transmisión de aproximadamente $10 \mathrm{cms}$. Al igual que IDRF, se basa en el concepto de inducción electromagnética que hace más rápido, seguro y sencillo el intercambio de datos basado en el paradigma del contacto entre el emisor y su posible objetivo Borrego-Jaraba, Ruiz, Gómez y Miguel (2011). Pero, a diferencia de IDRF, generalmente se usan dos dispositivos iguales (un iniciador y su objetivo), que pueden funcionar en ambos modos operativos. La combinación permite tres modos de aplicación: los dispositivos de NFC establecen una conexión bidireccional, o un dispositivo se usa para leer o escribir una etiqueta externa, o el dispositivo se utiliza como tarjeta inteligente. Las ventajas de esta tecnología son varias: con la NFC los usuarios de móviles pueden intercambiar datos e información de forma segura con otros dispositivos NFC y sus correspondientes etiquetas; no se requieren complicados acoplamientos como en el caso del bluetooth. Además, un dispositivo NFC puede funcionar pasivamente, por ejemplo, sin batería (Egger, 2013). 
Las tecnologías de geolocalización ayudan al pasajero, ya quelos nuevos mapas proporcionan a los visitantes la visión de la información de los lugares del aeropuerto tocando un botón. Incluyen todos los servicios del aeropuerto, puertas y mostradores de facturación, tiendas y otros puntos de interés.Recientemente se ha habilitado Google MapsIndoor en el Aeropuerto de Sydney. Otra de las características de los mapas ampliables es que incluyen cobertura tanto interior como exterior e integración con varios medios de transporte (Bates, 2017).

El surgimiento de la robótica ha ido a la par que el ascenso de la Inteligencia Artificial (IA). Ésta, junto con el aprendizaje de la máquina y la analítica predictiva, tiene multitud de aplicaciones en la industria aeroportuaria, como robots de etiquetado y facturación de equipaje, robots devigilancia y el chatbot- software diseñado para realizar una serie de tareas por su cuenta como reservas o conversar con los clientes y responder en tiempo real, de manera similar a la humana -.

De hecho es una de sus disciplinas, implantada ya. En el 2016 se convirtió en una tecnología útil para el cliente en las terminales con prototipos como el robot Spencer de KLM. En Tokio, el aeropuerto de Haneda ha anunciado el lanzamiento de su robot Experiment Project, que permitirá a empresas y a organizaciones de investigación probar la tecnología en el entorno del aeropuerto con 17 robots que harán ensayos en las áreas de limpieza, transporte y atención a los pasajeros. (Hosteltur, 2017).

"Internet ofThings" (IoT) o "Internet de las Cosas" no es, en realidad, una tecnología. El término fue acuñado por Kevin Ashton en 1999 (Ashton, 2009), que lo definió como una red que conecta todas las cosas en cualquier momento y en cualquier lugar para identificar, localizar, gestionar y monitorizar objetos objetos inteligentes. Zhou et al. (2015) y Zhou y Chao (2011) presentan un sistema de seguimiento de carga con una arquitectura basada en IoT. Cao, Jing y Zheng (2013) proponen y diseñan un un sistema de seguridad para el perímetro de un aeropuerto basado en IoT.

La tecnología más reciente, es sin duda, el blockchain.El 31 de Octubre de 2008 el libro blanco "Bitcoin - Un Sistema Peer to peer de Efectivo Electrónico" fue distribuido por un misterioso Satoshi Nakamoto a una lista de correo de criptógrafos(Popper, 2015).Esta tecnología está estrechamente asociada con la moneda virtual Bitcoin, pero su potencial va más allá. Esencialmente es un libro digital seguro de transacciones y acuerdos, ofrece "privacidad por diseño", por lo que los datos están seguros, cifrados, inviolables e inutilizables para cualquier otro propósito, y elimina la necesidad de una sola autoridad central. Permite trazar y autentificar personas y productos físicos gracias a técnicas de huellas, de reconocimiento numérico y de sensores. Puede tratarse, por ejemplo, del equipaje. Esta tecnologías hacen converger al mundo físico con el mundo numérico mejorando la trazabilidad de los productos y servicios (Waelbroeck, 2017). Sus posibilidades de aplicación aún están por explorar.

Existen sistemas que combinan estas tecnologías, como la de Alghadeir y Al-sakran (2016), que proponen una arquitectura informática para aeropuertos integrando tecnología RFID, IoT y Google Indoor, que incluye una aplicación para smartphone, kioscos, etiquetas para equipaje y lectores RFDI para distribuir éste a cintas de transporte específicas. Los kioscos ayudan al viajero a imprimir la tarjeta de embarque y las etiquetas para el 
equipaje. Todos estos datos estarán conectados a una base de datos para proveer los servicios del aeropuerto.

La operación aeroportuaria tiene que contemplar cuatro procesos diferentes relacionados con: los pasajeros, el equipaje, la carga y la aeronave (Carmona, 2004).En concreto, nos referimos a los procesos que realiza el pasajero hasta su entrada en el avión, esto es: embarque, obtención de etiquetas de equipaje, facturación del equipaje, control aduanero y ocio durante los tiempos de espera. Las tecnologías a las que antes nos referimos han posibilitado la instalación de distintos dispositivos como quioscos de embarque, tornos de acceso, máquinas de control biométrico de pasaportes, quioscos de impresión de etiquetas de equipaje, quioscos de entrega del equipaje, guía de realidad aumentada dentro del aeropuerto y videojuegos.

Estos dispositivos, llamados también Tecnologías Autoservicio (TA), puesto que son utilizados por el pasajero sin intervención de personal del aeropuerto, han recibido opiniones en contra por la ausencia del servicio cara a cara, valorado hasta ahora por el cliente. Efectivamente, las TA han dejado de lado a ese tipo de clientes, lo cualcon toda probabilidad, tendrá un impacto negativo en su experiencia de servicio. La cuestión es si la despersonalización del servicio tiene influencia en el uso del las TA en los aeropuertos.

Gibbs 2014 (en Otieno y Govender, 2016) concluyó que la despersonalización no tiene por qué afectar negativamente la satisfacción del pasajero, ya que los pasajeros valoran otros atributos como la fiabilidad, la eficiencia y el ahorro de tiempo de espera provistos por las plataformas autoservicio. Aunque la percepción de los encuestados acerca de la conveniencia de las TA indicaba una fuerte relación con el cumplimiento del servicio, sin embargo parecían haber dado más importancia relativa en las TA a la conveniencia que a la facilidad de uso o la fiabilidad. Es posible que los pasajeros hubieran visto la funcionalidad y la consistencia como un factor obvio, y que esto les interese más que las otras variables en el caso de estas tecnologías.

El Modelo clásico de Aceptación de la Tecnología (TAM) (Davis, 1985) postulaba que la aceptación de la tecnología depende fundamentalmente de la utilidad de la tecnología y la facilidad percibida de uso La Utilidad percibida es definida como "el grado en que una persona cree que el uso de un determinado sistema de información o tecnología mejorará su desempeño laboral". La Facilidad de uso percibida se refiere al "grado en que una persona cree que el uso de un sistema de información en particular está libre de esfuerzo" (Davis, 1989, p. 312) .

En esta misma línea, el estudio de Otieno y Govender realizado en el aeropuerto internacional O.R. Tambo (Johannesburgo, Sudáfrica), reveló que la percepción de eficiencia de la tecnología que el consumidor tiene está asociada a la comodidad, fiabilidad, y facilidad de uso de las tecnologías auto-servicio(Otieno y Govender, 2016). Así mismo, Gounaris afirma que la facilidad de uso de las tecnologías de la información determinan su adopción y utilización (Gounaris, Dimitriadis, y Stathakopoulos, 2010; Narteh, 2015).La empresa SITA lleva varios años realizando encuestas de satisfacción sobre tecnologías en 17 países de Europa, África, Asia, América y el Medio Este, pero no publica los resultados de cada país individualmente. En su informe del año 2017 concluye que la satisfacción de los pasajeros aumenta con el uso de las tecnologías (SITA, 2017). 
Deseamos conocer la aceptación de las nuevas tecnologías entre los pasajeros españoles, por tanto, una de las hipótesis a validar sería:

- $\mathrm{H}_{1}$ : La utilización de tecnología en el aeropuerto aumenta la satisfacción del pasajero

\subsection{Relación entre la edad del pasajero y su aceptación de la tecnología}

Diversos autores han estudiado la importancia de la edad en la aceptación de la tecnología, tanto de las generaciones X, Y y Z, como de los llamados "Millenials", o la "Generación C", postmillenials conectados, multicanal, ávidos de contenidos y dispuestos a mantener el control de los que ellos mismos crean y publican (Dimanche, 2010).En 2003 Venkatesh, Morris, Davis y Davis formularon un modelo llamado Teoría Unificada de Aceptación y Uso de la Tecnología (UTAUT), en la cual añadieron como variables explicativas de la aceptación tecnológica la influencia del entorno social, la posibilidad de acceso a la tecnología y la intención de uso; pero además, validaron la edad como moderador de las relaciones entre algunas de estas variables. En concreto, establecieron que la edad atenuaba las relaciones entre la expectativa de rendimiento y la intención de uso, el esfuerzo esperado y la intención de uso, la influencia social y la intención de uso y las condiciones de acceso a la tecnología y el uso(Venkatesh, Morris, Davis y Davis, 2003).

A partir de este momento, otros autores comoTorres, Robles y Molina (2011) sugieren que a los mencionados factores de aceptación de la tecnología habría que añadir otros de corte sociológico. En un trabajo posterior, concluyeron que el uso de Internet depende también de la edad, el nivel de estudios y las habilidades digitales. Señalan también, que es de esperar que la variable edad pierda protagonismo en el uso de Internet, en la medida en que estos jóvenes se conviertan en adultos o mayores del mañana (Torres, Robles, de Marco y Antino, 2017).

Otieno y Govender (2016) sugieren que sería interesante estudiar la actitud de las diferentes generaciones hacia las tecnologías autoservicio en las distintas fases de los procesos aeroportuarios, por ejemplo en el auto-etiquetado del equipaje.

En consecuencia, la segunda hipótesis que se desea probar es

- $\mathrm{H}_{2}$ : La edad está relacionada con la aceptación de las tecnologías en los aeropuertos

\section{METODOLOGÍA}

Para validar las hipótesis de trabajo se ha utilizado el método cuantitativo, concretamente la encuesta cara a cara autoadministrada.A partir de la información recopilada se elaboró un cuestionarioconteniendo:

(i) una pregunta filtro, para excluir a las personas que no hubieran viajado en avión en los dos últimos años,

(ii) 7 preguntas cerradas de tipo sociodemográfico,

(iii) 5 preguntas dicotómicas acerca de las tecnologías que desearían usar los pasajeros, 
(iv) 3 preguntas dicotómicas sobre las tecnologías que hubieran usado en su último viaje en los procesos de embarque, obtención de etiquetas de equipaje y facturación del equipaje,

(v) y 3 de escala tipo Likert de 5 puntos en las que 1 es la calificación más baja y 5 la más alta,sobre su satisfacción en cada uno de esos tres procesos.

La representatividad de la muestra queda garantizada por la alta tasa de respuesta (el $85 \%$ en la encuesta total).

\section{Tabla 1 \\ FICHA TÉCNICA DE LA ENCUESTA}

\begin{tabular}{ll}
\hline \multicolumn{2}{c}{ Características de la población y la muestra } \\
\hline Población objeto de estudio & $\begin{array}{l}\text { Españoles que hayan viajado en avión en los dos } \\
\text { útimos años }\end{array}$ \\
Ámbito geográfico & España \\
Recogida de la información & Encuesta cara a cara autoadministrada \\
Tratamiento de la información & Programa estadístico Statgraphics \\
Tamaño de la población & Superior a 10.000 (Infinita) \\
Tamaño de la muestra & 400 \\
Método & Muestreo Aleatorio Simple \\
Error muestral & $+/-5 \%$ \\
Nivel de confianza & $95 \%$ (p=q=0,5) \\
Periodo de recogida de la información & Abril -Mayo 2018 \\
Duración del cuestionario & $5-7$ minutos \\
Técnicas estadísticas utilizadas & Análisis descriptivo. Chi Cuadrado \\
\hline
\end{tabular}

Fuente: Elaboración propia.

El cuestionario se sometió a una prueba piloto los días 6 y 8 de marzo de 2018 en la Escuela Técnica Superior de Ingeniería Aeronáutica y del Espacio de la Universidad Politécnica de Madrid a 10 trabajadores de administración y servicios, 10 miembros del personal docente e investigador y 10 alumnos. En la Tabla 1 se muestra la ficha técnica de la encuesta.

La validez de contenido del cuestionario se obtiene con la revisión bibliográfica identificando las variables para el logro de los objetivos del estudio, derivando en las preguntas de la encuesta; la validez de criterio se comprueba en la evaluación de expertos y la prueba piloto.

La encuesta definitiva se realizó en los meses de Abril y Mayo de 2018, en las inmediaciones del Estadio Santiago Bernabéu de Madrid en las horas previas a los partidos. Se eligió este enclave por varias razones: 
- La población objetivo eran españoles que hubieran volado al menos una vez en los dos últimos años. Los aficionados al fútbol vienen de todas partes de España, y son usuarios frecuentes de avión, ya que muchos de los desplazamientos para asistir a partidos de sus equipos en otros países los realizan con este medio de transporte.

- En un aeropuerto, las respuestas del pasajero están condicionadas por esa última experiencia que han tenido y la situación emocional que estén viviendo en ese momento. En las horas previas a un evento deportivo es previsible que las opiniones expresadas sean producto de experiencias meditadas en el tiempo e interiorizadas.

- La tasa de respuesta se esperaba que fuera elevada, lo cual se cumplió; ya que en las horas previas a un evento deportivo los aficionados suelen estar relajados y tienen tiempo para responder.

Se contó con la ayuda adicional de cuatro encuestadores. La encuesta fue autoadministrada, pero los encuestadores contestaron las posibles dudas de los encuestados y cuidaron de que terminaran las encuestas, por tanto, la tasa de abandono fue inapreciable. De las 474 personas que completaron el cuestionario, 46 se descartaron por manifestar en la pregunta filtro no haber volado en los últimos dos años (un 15\%), quedando un total de 428 encuestas. De éstas, sedesecharon 28 (el 6,54\%) por no estar correctamente completadas. Por tanto, finalmente se analizaron 400 encuestas.

\section{RESULTADOS}

\subsection{Características básicas de la muestra}

Figura 1

\section{EDAD DE LAS PERSONAS ENCUESTADAS}

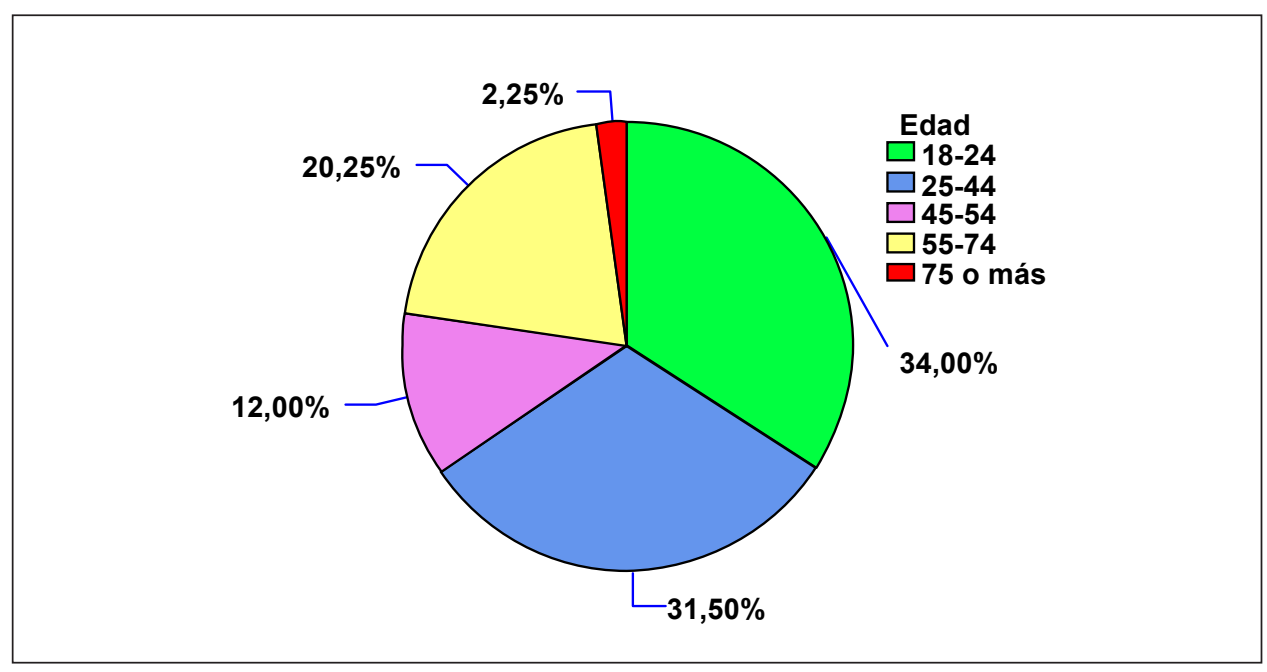

Fuente: Elaboración propia. 


\subsection{Satisfacción de los pasajeros}

Las respuestas relativas a la satisfacción de los encuestados en cada etapa de su último viaje, en relación con la forma en que habíanembarcado, obtenido las etiquetas de equipaje y facturado su equipaje (con tecnología o sin ella) se resumen en la tabla 2. Se observa que de los 400 encuestados, la media de satisfacción de los 89 que habían embarcado en persona fue de 3,33 sobre 5, mientras que los 311 que declararon haber embarcado mediante una app, por internet, o en un kiosco autoservicio, tuvieron una media de satisfacción de 3,67 sobre 5 .

Tabla 2

RESUMEN ESTADÍSTICO DE SATISFACCIÓN DE LOS PASAJEROS

\begin{tabular}{|c|c|c|c|c|c|c|}
\cline { 2 - 7 } \multicolumn{1}{c|}{} & \multicolumn{2}{c|}{ Embarque } & \multicolumn{2}{c|}{ Etiquetas de equipaje } & \multicolumn{2}{c|}{ Facturación } \\
\cline { 2 - 7 } & Personal & $\begin{array}{c}\text { Con } \\
\text { tecnología }\end{array}$ & $\begin{array}{c}\text { En } \\
\text { mostrador }\end{array}$ & $\begin{array}{c}\text { Mediante } \\
\text { autoservicio }\end{array}$ & $\begin{array}{c}\text { En } \\
\text { mostrador }\end{array}$ & $\begin{array}{c}\text { Mediante } \\
\text { autoservicio }\end{array}$ \\
\hline Recuento & 89 & 311 & 212 & 70 & 254 & 28 \\
\hline $\begin{array}{c}\text { Promedio de } \\
\text { satisfacción }\end{array}$ & 3,32584 & 3,67524 & 3,38679 & 3,84286 & 3,20472 & 4,21429 \\
\hline $\begin{array}{c}\text { Desviación } \\
\text { Estándar }\end{array}$ & 1,01997 & 0,970865 & 0,876991 & 0,773393 & 0,984774 & 1,03126 \\
\hline $\begin{array}{c}\text { Coeficiente de } \\
\text { Variación }\end{array}$ & $30,6681 \%$ & $26,4164 \%$ & $25,8945 \%$ & $20,1255 \%$ & $30,7288 \%$ & $24,4705 \%$ \\
\hline $\begin{array}{c}\text { Sesgo } \\
\text { Estandarizado }\end{array}$ & $-1,16336$ & $-4,99782$ & 1,57788 & $-1,02136$ & 1,33351 & $-2,88602$ \\
\hline $\begin{array}{c}\text { Curtosis } \\
\text { Estandarizada }\end{array}$ & 0,375932 & 1,12111 & $-1,75154$ & $-0,296528$ & $-1,50941$ & 0,851411 \\
\hline
\end{tabular}

Fuente: Elaboración propia.

En la figura 2 se muestra la media de satisfacción de los usuarios de tecnología en cada uno de los procesos estudiados, frente a la media de satisfacción de los no usuarios. Se aprecia una mayor satisfacción de los usuarios de tecnología en los tres procesos, más acusada en la facturación delequipaje.Si bien en el proceso de embarque la diferencia es muy pequeña, tan sólo un $8,4 \%$, entendemos que es positiva y por tanto se valida la hipótesis 1 :

$\mathrm{H}_{1}$ : La tecnología aumenta la satisfacción de los pasajeros 


\section{Figura 2 \\ SATISFACCIÓN DE LOS PASAJEROS}

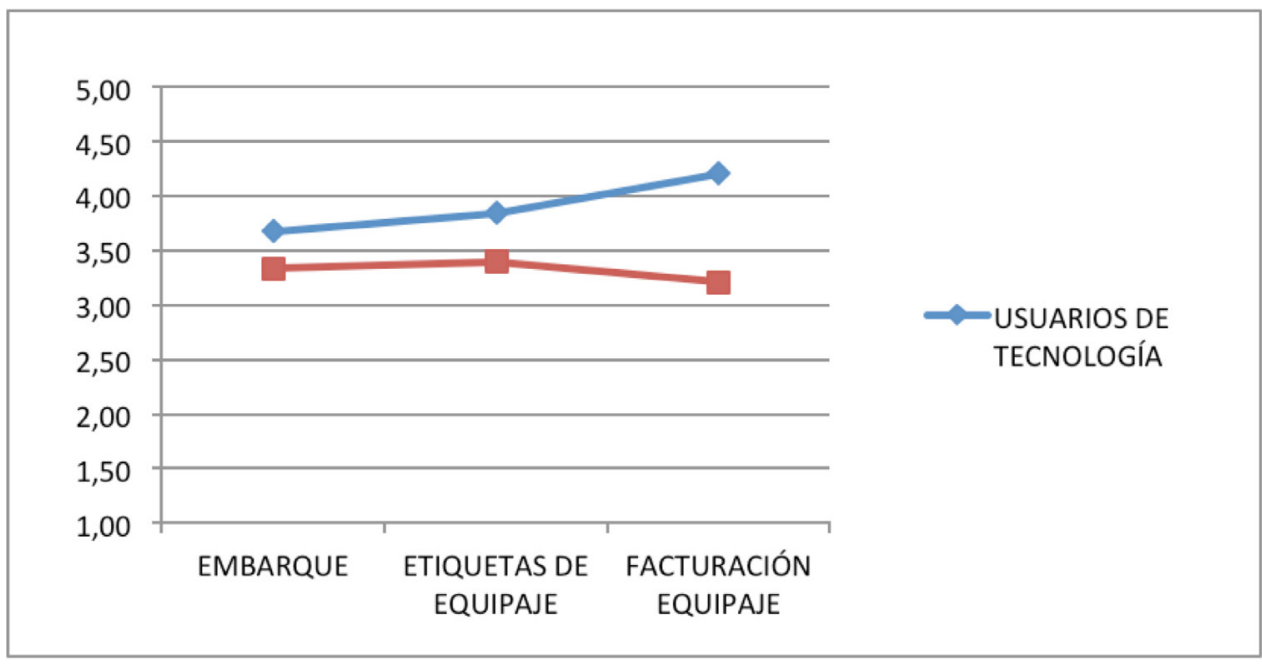

Fuente: Elaboración propia.

\subsection{Relación entre la edad y la aceptación de las tecnologías en los aeropuertos}

En la figura 3 se muestran los resultados de la encuesta en cuanto a los deseos de uso de tecnología. El 67,75\% manifestaron que desearían utilizar el pasaporte biométrico en su próximo viaje, el $60,75 \%$ desearían usar un servicio de realidad aumentada para guiarse por el aeropuerto y el $84 \%$ desearían embarcar con tecnologías autoservicio (app, internet o kiosco autoservicio), en vez de hacerlo personalmente.

El $60 \%$ desearía imprimir las etiquetas de su equipaje en casa o en el aeropuerto en un puesto autoservicio frente al $40 \%$ que desean seguir haciéndolo en el mostrador de la aerolínea. Menor aceptación presenta el uso de videojuegos, ya que tan sólo el 18,75\% manifiestan que utilizarían videojuegos mientras esperan, si se lesproporciona el servicio a un precio razonable. De la misma manera, el 30,25\% desearía facturar su equipaje en un puesto autoservicio y no en el mostrador de la aerolínea.

Para validar la segunda hipótesis, se han construidoseis tablas de contingencia ente la variable edad distribuida en los cinco grupos expuestos anteriormente y cada una de las variables "Deseo de uso de pasaporte biométrico en su próximo viaje", "Deseo de videojuegos en el aeropuerto", "Deseo de un servicio de realidad aumentada gratuito en su smartphone para guiarse por el aeropuerto", "Deseo de usar tecnologías autoservicio en su próximo embarque", "Deseo de usar tecnologías autoservicio para la obtención de etiquetas de equipaje en su próximo viaje" y "Deseo de facturar mediante tecnologías autoservicio en su próximo viaje", y se han efectuado los correspondientes contrastes de independencia Chi-cuadrado. En la tabla 3 se resumen los resultados de los seis contrastes de independencia realizados. 


\section{Figura 3 \\ DESEOS DE USO DE TECNOLOGÍA}

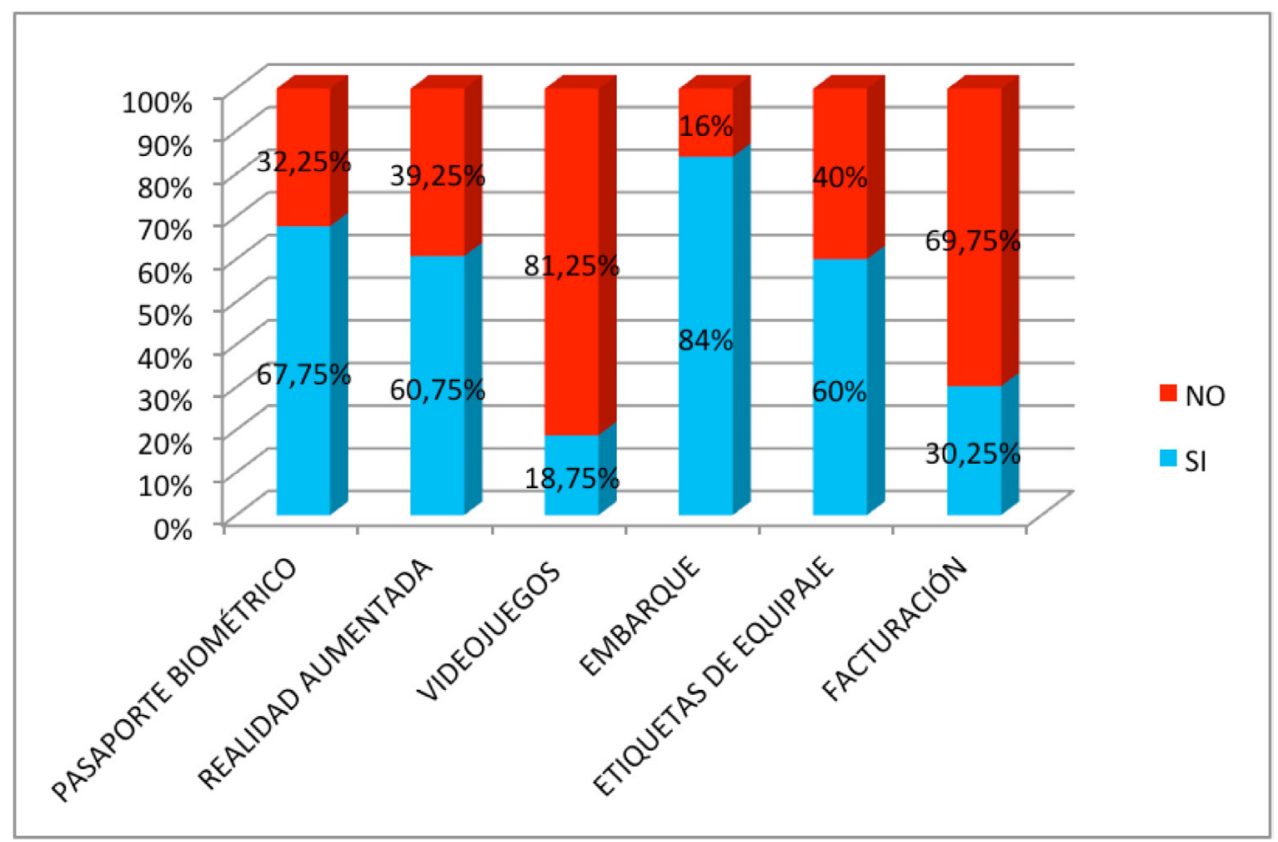

Fuente: Elaboración propia.

Tabla 3

CONTRASTES DE INDEPENDENCIA CHI- CUADRADO EDAD PARA DESEOS DE TECNOLOGÍA

\begin{tabular}{|l|c|c|c|c|c|c|}
\hline & $\begin{array}{l}\text { Pasaporte } \\
\text { Biométrico }\end{array}$ & Videojuegos & $\begin{array}{l}\text { Servicio de } \\
\text { Realidad } \\
\text { Aumentada }\end{array}$ & Embarque & $\begin{array}{l}\text { Etiquetas } \\
\text { de equipaje }\end{array}$ & Facturación \\
\hline Estadístico & 17,291 & 9,655 & 3,196 & 36,163 & 26,268 & 39,489 \\
\hline Gl & 4 & 4 & 4 & 4 & 4 & 4 \\
\hline Valor-P & 0,0017 & 0,0467 & 0,5255 & 0,0001 & 0,0002 & 0,0001 \\
\hline $\begin{array}{l}\text { Se rechaza } \\
\text { independencia }\end{array}$ & SI & SI & NO & SI & SI & SI \\
\hline
\end{tabular}

Fuente: Elaboración propia.

En estos contrastes se rechaza la hipótesis de independencia entre las variableal $95 \%$ de confianza, si el P-valor es menor que 0,05.Como se puede observar en la Tabla 3, tan sólo la variable "Deseo de servicio de Realidad Aumentada en el Aeropuerto" ha resultado ser independiente de la edad.Por tanto, validamos esta segunda hipótesis: 
- $\mathrm{H}_{2}$ : La edad está relacionada con la aceptación de las tecnologías en los aeropuertos, tal como habían observado otros autores en estudios similares llevados a cabo fuera del ámbito aeroportuario (Torres et al., 2017; Torres et al., 2011).

Tabla 4

SATISFACCIÓN POR EDADES

\begin{tabular}{|l|l|l|l|l|l|l|l|l|l|l|l|}
\hline \multicolumn{4}{|l|}{ Embarque } & \multicolumn{4}{l|}{ Etiquetas } & \multicolumn{3}{l|}{ Facturación } \\
\hline $18-24$ & $25-44$ & $45-54$ & 55 o más & $18-24$ & $25-44$ & $45-54$ & 55 o más & $18-24$ & $25-44$ & $45-54$ & 55 o más \\
\hline 3,75 & 3,69 & 3,38 & 3,68 & 3,83 & 3,86 & 3,86 & 3,83 & 4,25 & 4,25 & 5 & 3,91 \\
\hline 3,21 & 2,9 & 3,67 & 3,28 & 3,35 & 3,38 & 3,54 & 3,4 & 3,19 & 3,19 & 3,23 & 3,41 \\
\hline
\end{tabular}

Fuente: Elaboración propia.

Al analizar la satisfacción de los pasajeros en los distintos procesos (embarque, etiquetas, facturación) según su edad, se observa una tendencia similar en los cuatro grupos de edad en cuanto a que la satisfacción tras haber usado tecnología es mayor en la facturación del equipaje, seguido del etiquetado del equipaje, y por último en el embarque. En la figura 4 podemos apreciar que el grupo de edad que experimentó mayor satisfacción tras el uso de tecnología en el proceso de facturación es el 45 a 54 años, en cambio, en los otros dos procesos este grupo se mostró más satisfecho al haberlos efectuado personalmente.

Figura 4

\section{SATISFACCIÓN POR EDADES}

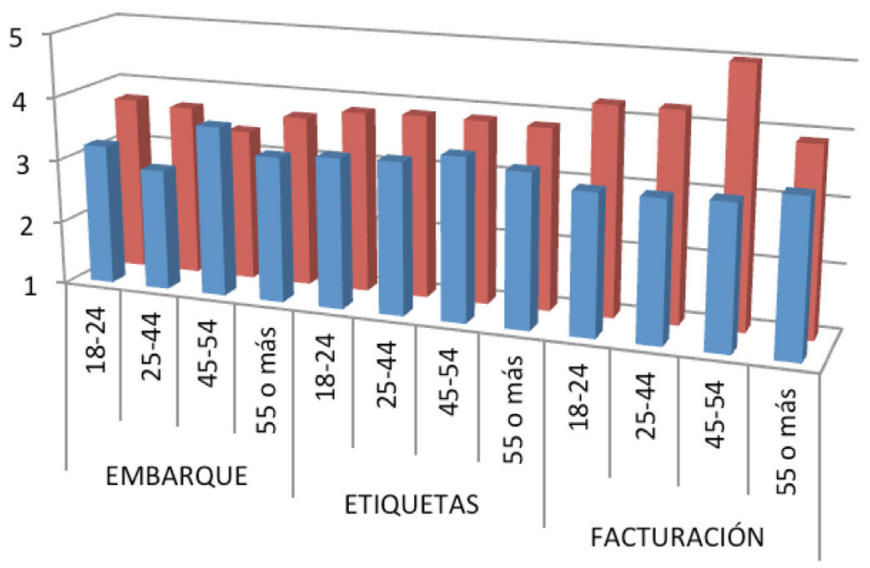

SIN TEC.

- CON TEC.

Fuente: Elaboración propia. 


\section{CONCLUSIONES}

El turismo en España tiene una importancia creciente, y es una parte fundamental de la actividad económica. El Aeropuerto se configura como una parte fundamental de la Ciudad Inteligente. Internet de las Cosas, la Comunicación de Campo Cercano, latecnología de Código de Barras, la Identificación por Radiofrecuencia, las tecnologías de geolocalización, la Realidad inmersiva, los sistemas biométricos, la Inteligencia Artificial y la Robótica y el Blockchain o Tecnología de Bloques son tecnologías que han permitido la creación de distintos dispositivos que ayudan al pasajero en todas las etapas de su viaje, facilitan su paso por el aeropuerto y lo convierten en "Aeropuerto Inteligente".

Los aeropuertos también juegan un papel estratégico para las regiones en las que operan, en cuanto a que pueden mejorar la visibilidad de un destino. La posición distintiva de un aeropuerto puede convertirse en un valor principal para la atracción de nuevas actividades de producción en ciertas zonas geográficas, y por tanto, un motor principal en la oferta de valor de un lugar. De hecho, las infraestructuras de transporte son lo primero que evalúan las multinacionales en el caso del desarrollo de actividades internacionales debido al impacto directo en la efectividad y la eficiencia.La implementación de estas tecnologías en los aeropuertos corresponde a entidades públicas, como la autoridad aeroportuaria y privadas, como las aerolíneas.

En este trabajo se ha planteado que estas tecnologías aumentaran la satisfacción del pasajero en las distintas etapas de su viaje y que la edad estuviera relacionada con la aceptación de la tecnología entre usuarios de avión españoles.Nuestro estudio demuestra que la satisfacción de los pasajeros que utilizaron la tecnología en los procesos de embarque, obtención de etiquetas de equipaje y facturación es mayor que la de aquellos que no las utilizaron,siendo esta relación mayor en la facturación de equipaje. Además, se ha podido confirmar que la edad está relacionada con los deseos de uso de dispositivos autoservicio en estos procesos y del pasaporte biométrico y videojuegos. El hecho de que el deseo de acceder a un servicio de realidad aumentada para guiarse por el aeropuerto haya resultado ser la única variable independiente de la edad de todas las estudiadas, puede llevarnos a pensar que las personas de más edad valoran muy positivamente la ayuda a ubicarse en lugares grandes que les resultan confusos.

La consecuencia lógica de este resultado es que las nuevas tecnologías de los aeropuertos inteligentes son ya un elemento competitivo en el sector de servicios aeroportuarios, pero que su total implantación depende de dos factores relacionados: el relevo de los pasajeros de más edad por los más jóvenes y la incorporación de los pasajeros de más edad a las nuevas tecnologías. El relevo mencionado tiene carácter demográfico y difícilmente se puede acelerar o frenar. La incorporación de los pasajeros de más edad a las nuevas tecnologías en parte no se producirá y en otra parte requerirá tiempo y acciones de difusión y promoción.

Los resultados obtenidos pueden servir de guía a los actores implicados (gestores de aeropuertos, aerolíneas y responsables políticos) en la decisión acerca de qué inversiones abordar. Como futuras líneas de investigación, los autores sugerimos un estudio más detallado de la aceptación del pasajero español de los distintos dispositivos del Aeropuerto Inteligente. 


\section{BIBLIOGRAFÍA}

ACI. (2005): «The Application of Biometrics at Airports». Position Paper. Airports Council International, 6 de noviembre de 2005. Disponible en http://www.aci.aero/Media/ aci/file/Free docs/ACI Biometric Position FINAL.pdf

ACI. (2009). «Manual de Políticas y Métodos Recomendados». Airports Councillnternational, 1 de noviembre de 2005. Disponible en http://www.aci.aero/Media/aci/file/ Publications/2009/Manual_de_Politicas_y_Metodos_Recomendados_de_ACI_2009_ v2.pdf

AENA (Asociación Española de Navegación Aérea) (2018): Transporte aéreo y turismo. Disponible en: http://www.aena.es/es/corporativa/transporte-aereo-y-turismo.html

ASHTON, K. (2009). That «Internet of Things» Thing. RFID Journal. Disponible en: http://www.rfidjournal.com/articles/view?4986

AUGÉ, M. (1992): Non-lieux. Introduction à uneanthropologie de la surmodernité. Edition de Seuil.

AVELAR, L. y SANTOS, B. (2014): «Trends - Augmented Reality», Journal Imagine, $\mathrm{n}^{\mathrm{o}} 2$.

BITNER, M.J. (1992): «Servicescapes: the impact of physical surroundings on customers and employees», Journal of Marketing, vol. 56 (2), pp. 57-71.

BORREGOJARABA, F., RUIZ, I. y GÓMEZ, N. (2011): «A NFC-pervasive solution for city touristic surfing», Personal and Ubiquitous Computing, vol. XV (7), pp. 731-742.

BOULANGER, N. y JENANNIN, H. (2016): «Une ville intelligente et humaine», Responsabilité \& Environnement, $\mathrm{n}^{\mathrm{O}} 4$, pp. 22-26.

BUHALIS, D. y AMARANGGANA, A. (2013): «Smart Tourism Destination», Information and Communication Technologies in Tourism 2014, pp. 553-564.

CAMISÓN, C., BOU, J.C., ROCA, V. y MONTESINOS, C. (1997): «Enlace de la investigación en turismo y en gestión de la calidad: balance de una década», Papers de Turisme, $\mathrm{n}^{\circ}$ 20, pp. 19-38.

CAO, X.M., JING, C. y ZHENG, X.D. (2013): «Design of the Airport Perimeter Security System Based on Internet of Things», Applied Mechanics and Materials, vols. 361, pp. 2.276-2.281.

CARAGLIU, A., DEL BO, C. y NIJKAMP, P. (2011): «Smart Cities in Europe», Journal of Urban Technology, ${ }^{\circ} 18$, pp. 65-82.

CARMONA, A.I. (2004): Servicios Aeroportuarios. Madrid, Fundación Aena.

CEYLAN, H., GOPALAKRISHNANAN, K. y KIM, S. (2015): «Smart airport pavement heath monitoring», International Airport Review, vol. XIX (3).

CHAU, V. y KAO, Y.-Y. (2009): «Bridge over troubled water or long and winding road?: Gap-5 in airline service quality performance measures», Managing Service Quality: An International Journal, vol. XIX, $\mathrm{n}^{\circ}$ 1, pp. 106-134.

CHEN, H. (2002): «Benchmarking and quality improvement: A quality benchmarking deployment approach», International Journal of Quality \& Reliability Management, vol. XIX (6), pp. 757-773.

CHUKHRAY, N. (2012): «Marketing Strategies for Overcoming Consumer Resistance to Innovations», Konsumpcja i Rozwój, vol. 1 (2), pp. 71-84. 
COHEN, B. (2012): «What exactly is a smart city?», en FastCompany, 19 de septiembre de 2012. Disponible en http://www.fastcoexist.com/1680538/what-exactly-is-a-smartcity

DAVIS, F. (1985): A technology acceptance model for empirically testing new end-user information systems: Theory and results. (Tesis doctoral). Massachusetts Institute of Technology.

DAVIS, F. (1989): «Perceived Usefulness, Perceived Ease Of Use, And User Acceptance of Information Technology», MIS Quarterly, vol. 13 (3), pp. 319-340.

DIMANCHE, F. (2010): «En quête de la «génération C». Pour un nouvel agenda de recherche marketing et tourisme», Mondes du Tourisme, $\mathrm{n}^{\circ} 1$, pp. 30-38.

EGGER, R. (2013): «The impact of near field communication on tourism», Journal of Hospitality and Tourism Technology, vol. 4 (2), pp. 119-133.

EGUÍA, B. y ALONSO, I. (2002): "El Desarrollo de las Tecnologías de la Información y la Comunicación: un nuevo reto para el mercado de trabajo", en Scripta Nova, vol. VI (119), 1 de octubre de 2002. Universidad de Barcelona. Disponible en http://www. ub.edu/geocrit/sn/sn119-74.htm

FLOM, L. y SAFFIR, A. (1985): Iris Recognition System, WO Patent, n ${ }^{\circ}$ 86/05018.

FLORIDOBENÍTEZ , L. (2016): «Mobile Apps : Improve Airports ' Brand Image and Differentiate Among Competitors», Journal of Tourism Research, vol. 6 (1), pp. 39-53.

FODNESS, DALE y MURRAY, B. (2007): «Passengers' expectations of airport service quality», Journal of Services Marketing, vol. 21 (7), pp. 492-506.

FORSYTH, P., GILLEN, D., MUELLER, J. y NIEMEIER, H.M. (2010): Airport Competition. The European Experience. Farnham, Ashgate Publishing.

GILBERT, D. y WONG, R.K.C. (2003): «Passenger expectations and airline services: A Hong Kong based study», Tourism Management, vol. 24 (5), pp. 519-532.

GOUNARIS, S., DIMITRIADIS, S. y STATHAKOPOULOS , V. (2010): «An examination of the effects of service quality and satisfaction on customers' behavioral intentions in e-shopping», Journal of Services Marketing, vol. 24 (2), pp. 142-156.

GRAHAM, A. (2013): Managing Airports. An international perspective. London, Taylor \& Francis Group.

GUTIÉRREZ, M., VEXO, F. y THALMANN, D. (2008): Stepping into Virtual Reality. Lausanne, Springer.

GUTTENTAG, D.A. (2010): «Virtual reality: Applications and implications for tourism», Tourism Management, vol. 31 (5), pp. 637-651.

HA, J., PAGE, T. y THORSTEINSSON, G. (2011): «A Study on Technophobia and Mobile Device Design», International Journal of Contents, vol. 7 (2), pp. 17-25.

HALL,C.M. (2009):El turismo como ciencia social de la movilidad. Madrid, Síntesis.

HOSTELTUR (2017): «Siete tecnologías revolucionarán la Industria de la Aviación». Revista mensual, Abril 2017.

HUSTON, S. (2015): The aerotropolis - development catalyst or distraction ?, en ResearchGate, 1 de mayo de 2015. Disponible en:

https://www.researchgate.net/publication/276895631_The_aerotropolis_-_development_ catalyst_or_distraction/download 
HUANG, C.D., GOO, J., NAM, K., y YOO, C.W. (2017): «Smart tourism technologies in travel planning: The role of exploration and exploitation», Informationand Management, vol. 54 (6), pp. 757-770.

IATA (International Air Transport Association) (2017): World Air Transport Statistics 2017. Disponible en: http://www.iata.org/pressroom/media-kit/Documents/WATS2017-mediakit-summary.pdf

IDC (2012): Smart Cities Analysis in Spain 2012, en IDC White papers, 1 de septiembre de 2012. Disponible en:

http://www.portalidc.com/resources/white_papers/IDC_Smart_City_Analysis_Spain_ EN.pdf

INGLADA, V.,COTO-MILLÁN, P. e INGLADA-PÉREZ, L. (2017): «Evaluación de la productividad y eficiencia en los aeropuertos españoles después de la liberalización del transporte aéreo», Revista de Evaluación de Programas y Políticas Públicas, $\mathrm{n}^{\circ}$ 9, pp. 99-112.

KASARDA, J.D. (2000): «Logistics \& the Rise of the Aerotropolis», Real Estate Issues, vol. 25 (4), pp. 43-48.

KOMNINOS, N., PALLOT, M. y SCHAFFERS, H. (2013): «Special Issue on Smart Cities and the Future Internet in Europe», Journal of the Knowledge Economy, vol. 4 (2), pp. 119-134.

KOO, C.; SHIN, S., GRETZEL, U. y NAMHO, C. (2016): «Conceptualization of Smart Tourism Destination Competitiveness», Asia Pacific Journal of Information Systems, vol. 26 (4), pp. 561-576.

LEE-MORTIMER, A. (1993): «Customer focus takes off», The TQM Magazine, vol. V (3), pp. 37-41.

LIOU, J.J., TANG, C.H., YEH, W.C. y TSAI, C.Y. (2011): «A decision rules approach for improvement of airport service quality», Expert Systems with Applications, vol. 38 (11), pp. 13.723-13.730.

MILGRAM, P., TAKEMURA, H., UTSUMI, A. y KISHINO, F. (1994): «Augmented reality: a class of displays on the reality-virtuality continuum», SPIE, vol. 2351, pp. 282-292.

NAM, T. y PARDO, T.A. (2011): «Conceptualizing Smart City with Dimensions of Technology , People and Institutions», The Proceedings of the 12th Annual International Conference on Digital Government Research, pp. 282-291. http://doi. org/10.1145/2037556.2037602

NARTEH, B. (2015): «Perceived service quality and satisfaction of Self-service technology», International Journal of Quality and Reliability Management, vol. 32 (4), pp. 361-380.

OACI (2015): «Machine Readable Travel Document», International Civil Aviation Organization, DOC Series No. 9303, Part 3. Disponible en: https://www.icao.int/publications/ Documents/9303_p3_cons_en.pdf

OTIENO, P. y GOVENDER, K. (2016): «Managing airport service quality. The impact of self-service technologies», Investment Management and Financial Innovations, vol. 13 (3), pp. 387-393. 
PANTOUVAKIS, A. y RENZI, M. F. (2016): «Exploring different nationality perceptions of airport service quality». Journal of Air Transport Management, vol. 52, pp. 90-98. PARASURAMAN, A., BERRY, L. y LEONARD, L. (1985): «A Conceptual Model of Service Quality and its Implication for Future Research (SERVQUAL)», Journal of Marketing, vol. 49 (4), pp. 41-50.

POPPER, N. (2015): Digital gold - Bitcoin and the inside story of the misfits and the millionaires trying to reinvent money. New York: Harper Collins.

REBELO, F., NORIEGA, P. y DUARTE, E. (2012): «Using Virtual Reality to Assess User Experience», Human Factors: The Journal of the Human Factors and Ergonomics Society, vol. 54 (6), pp. 964-982.

RENDEIRO, R. (2006): «Tourism service quality begins at the airport», Tourism Management, vol. 27 (5), pp. 874-877.

RHOADES, D.L., BAGUESPACK, B. y YOUNG, S. (2000): «Developing a quality index for US airports», Managing Service Quality: An International Journal, vol. 10 (4), pp. 257-262.

ROBLEDO, M.A. (1997). «Una aplicación del modelo Servqual de calidad de servicio a la industria del transporte aéreo», Papers de Turisme, $\mathrm{n}^{\circ}$ 20, pp. 117-135.

RUIZ, B. y PINDADO,S. (2012): Transporte Aéreo. Madrid, Universidad Politécnica de Madrid.

SEGITTUR. (2015): Informe destinos turísticos inteligentes: construyendo el futuro. Madrid, Ministerio de Industria, Energía y Turismo.Disponible en: http://www.segittur.es/es/DTI/dti-detalle/Libro-Blanco-Destinos-Tursticos-Inteligentes-/\#.Wmy0EZOdVsM.

SEIDEMAN, T. (1993). «Bar codes sweep the world», Invention and Technology Magazine.

Disponible en: http://www.americanheritage.com/articles/magazine/it/1993/4/1993_4_56. shtml

SERRANO, J.M., ESPEJO, C. y GARCÍA, R. (2016): “Accesibilidad aérea en la Región de Murcia: una encrucijada compleja desde una perspectiva turística", Papers de Turisme, $\mathrm{n}^{\circ}$ 59, pp. 62-85.

SINKOVICS , R. R., STÖTTINGER, B., SCHLEGELMILCH, B.B. y RAM, S. (2002): «Reluctance to use technology-related products: Development of a technophobia scale». Thunderbird International Business Review, vol. 44 (4), pp. 477-494.

SITA. (2017). «The Passenger IT Trends Survey». Air Transport World. Disponible en : http://www.sita.aero/socialhub

SULTAN, F. y SIMPSON, M. (2000): «International service variants: airline passenger expectations and perceptions of service quality», Journal of Services Marketing, vol. 14, pp. 188-216.

TAJIMA, M. (2007): «Strategic value of RFID in supply chain management», Journal of Purchasing and Supply Management, vol. 13 (4), pp. 261-273.

TORRES, C., ROBLES, J.M., DE MARCO, S. y ANTINO, M. (2017): «Revisión analítica del modelo de aceptación de la tecnología. El cambio tecnológico», Papers, vol. 102 (1), pp. 5-27. 
TORRES, C., ROBLES, J.M. y MOLINA, O. (2011): «¿Por qué usamos las tecnologías de la información y las comunicaciones? Un estudio sobre las bases sociales de la utilidad individual de internet», Revista Internacional de Sociología, vol. 69 (2), pp. 371-392.

TSAI, W.H., HSU, W. y CHOU, W.C. (2011): «A gap analysis model for improving airport service quality», Total Quality Management \& Business Excellence, vol. XXII (2), pp. $1.025-1.040$.

VANOLO, A. (2013): «Smartmentality: The Smart City as Disciplinary Strategy», Urban Studies, vol. 51 (5), pp. 883-898.

VENKATESH, V., MORRIS, M., DAVIS, G. y DAVIS, F. (2003): «User acceptance of information technology: Toward a unified view», MIS Quarterly, vol. 27 (3), pp. 425 478.

VERGARA, A. y DE LAS RIVAS, J.L. (2004): Territorios inteligentes. Madrid, Fundación Metrópoli.

WAELBROECK, P. (2017): «Les enjeux économiques de la blockchain», Annales des Mines - Réalités industrielles, $\mathrm{n}^{\mathrm{o}} 3$, pp. 10-19.

WOODLAND, N.J. y BERNARD, S. (1952): Classifying apparatus and method, U.S. Patent, $n^{\circ} 2,612,994$.

YEH, C.H. y KUO, Y.L. (2003): «Evaluating passenger services of Asia-Pacific international airports», Transportation Research Part E: Logistics and Transportation Review, vol. XXXIX, (1), pp. 35-48.

ZHOU, L., CHONG, A. y NGAI, E.W.T. (2015): "Supply chain management in the era of the internet of things", International Journal of Production Economics, vol. 159, pp. 1-3.

ZHOU, L. y CHAO, H.-C. (2011): "Multimedia traffic security architecture for the internet of things", IEEE Network, vol. 25 (3), pp. 35-40. 
